\title{
The Maximum Effect of an Increase in Rate on Human Ventricular Refractoriness
}

\author{
FRED MORADY, ALAN H. KADISH, LAURI K. TOIVONEN, \\ JEFFREY A. KUSHNER, and STEPHEN SCHMALTZ
}

From the Division of Cardiology and the Clinical Research Center, Department of Internal Medicine, University of Michigan Medical Center, Ann Arbor, MI

MORADY, F., ET AL.: The maximum effect of an increase in rate on human ventricular refractoriness. The purpose of this study was to determine the maximum shortening of ventricular refractoriness that occurs following an increase in rate and to quantitate the duration of ventricular pacing required to obtain this maximum shortening of refractoriness. The subjects of the study consisted of 41 patients who underwent a clinically indicated electrophysiologic study. Ventricular refractory periods were measured with an extrastimulus $\left(S_{2}\right)$ at basic cycle lengths of 600 and $400 \mathrm{~ms}$ by Method $A$ ( 8 beat basic drive trains and 4 second intertrain paue and Method $B$ (drive train duration of 3 minutes, then an $S_{2}$ after every eighth basic drive beat, with no pause after the $S_{2}$ ). In 23 subjects, the mean ventricular effective refractory period determined by Method $B$ was $12 \pm 7 \mathrm{~ms}$ ( \pm standard deviation) shorter than when determined by Method $A$ at a basic drive cycle length of $600 \mathrm{~ms}(\mathrm{p}<0.0001)$ and $33 \pm 9 \mathrm{~ms}$ shorter at a basic drive cycle length of 400 $\mathrm{ms}(\mathrm{p}<0.001)$. In these 23 subjects, the drive train duration required for maximum shortening of ventricular refractoriness was estimated by counting the number of drive train beats preceding ventricular capture by an $S_{2}$ inserted after every fourth basic drive beat at a coupling interval fixed at 5 ms longer than the ventricular effective refractory period determined in that subject by Method $B$. The mean number of basic drive beats preceding capture by $S_{2}$ was $114 \pm 84$ beats at a basic drive cycle length of $600 \mathrm{~ms}$ and $233 \pm 85$ beats at a BDCL of $400 \mathrm{~ms}$. In six subjects the ventricular effective refractory period was measured by Methods $A$ and $B$ before and after autonomic blockade with propranolol and atropine, and the amount of shortening in the ventricular effective refractory period with Method B was not affected by autonomic blockade. In conclusion, the basic drive train has a cumulative effect on ventricular refractoriness in humans, and a drive train duration substantially longer than 50 beats often is required to obtain the maximum shortening of ventricular effective refractory period after an increase in rate. Therefore, ventricular effective refractory periods determined conventionally using 8 beat drive trains and a 4 second intertrain pause often may be overestimates of the actual ventricular effective refractory period. The shortening of ventricular refractoriness with long drive train durations is probably related to a prolonged duration of pacing required to obtain a steady-state action potential duration after an increase in rate. (PACE, Vol. 11, December 1988)

ventricular refractoriness, autonomic blockade, drive train duration

\section{Introduction}

The action potential duration and refractory period of ventricular muscle are inversely related

Supported in part by Grants 5M01-RR00042-25 and 1R29 HL40667-01 from the National Institutes of Health, Bethesda, MD.

Address for reprints: Fred Morady, M.D., Division of Cardiology, University Hospital, 1500 E. Medical Center Drive, UH B1 F2456/0022, Ann Arbor, MI 48109-0022.

Received May 17, 1988; revised July 11, 1988; accepted July 12 , 1988. to rate. ${ }^{1-4}$ Although several experimental studies have demonstrated that up to several minutes may be required to obtain the maximum decrease in action potential duration and ventricular refractoriness when the stimulation rate increases, ${ }^{1,5-10}$ scant data are available regarding the time course of changes in ventricular refractoriness following an increase in rate in humans. Preliminary studies in humans have demonstrated that the ventricular effective refractory period progressively shortens as the drive train duration increases from 1 beat to 50 beats. ${ }^{1,12}$ However, 
based on the time course of changes in action potential duration and ventricular refractoriness in experimental studies, it is possible that a drive train duration longer than 50 beats is required to obtain the maximum shortening of ventricular refractoriness after an increase in rate.

The purpose of the present study was to determine the maximum degree of shortening in human ventricular refractoriness that occurs following an increase in rate and to quantitate the duration of ventricular pacing required to obtain this maximum degree of shortening in ventricular refractoriness.

\section{Methods}

\section{Study Design}

Several experimental studies utilizing isolated ventricular muscle preparations obtained from dogs, cats, and rabbits have demonstrated that 1 to 3 minutes are generally needed for the ventricular muscle action potential to stabilize following a change in stimulation rate.$^{1,6-10}$ Therefore, it was hypothesized that 3 minutes of continuous ventricular pacing would be sufficient to obtain the maximum shortening in ventricular refractoriness that accompanies an increase in rate in humans. In the first part of this study, the use of a 3 minute drive train duration was validated by comparing the ventricular effective refractory period determined after 3 minutes of continuous pacing at a cycle length of 600 or $400 \mathrm{~ms}$ with the ventricular effective refractory period determined following 10 minutes of continuous pacing at the same cycle length. It was presumed that 10 minutes of pacing at a constant rate was long enough to ensure that the maximum effect of an increase in rate on ventricular refractoriness had been obtained.

In the second part of the study, the maximum effect of an increase in rate on ventricular refractory periods was determined by comparing the ventricular effective and functional refractory periods measured by two methods. The first method (Method A) was a conventional extrastimulus technique for measuring refractory periods using 8 beat drive trains and a 4 second intertrain pause. ${ }^{13-18}$ The second method (Method B) used 3 minutes of continuous ventricular pacing at the basic drive cycle length before introduction of an extrastimulus every eighth beat, with no pause after the extrastimulus. The rationale for not using a pause following the extrastimulus in Method B was to avoid disrupting the steady-state ventricular muscle action potential duration that was presumed to have been achieved after $3 \mathrm{~min}$ utes of continuous pacing.

The third part of this study was designed to estimate the duration of pacing required to obtain the maximum shortening in ventricular refractoriness after the onset of ventricular pacing at cycle lengths of 600 and $400 \mathrm{~ms}$. This was accomplished by counting the number of basic drive beats preceding ventricular capture by an extrastimulus positioned just beyond the effective refractory period as determined by Method $B$.

The purpose of the last part of this study was to assess the influence of the autonomic nervous system on the difference in ventricular refractory periods as determined by Methods A and B. Refractory periods were measured with Methods A and $B$ before and after autonomic blockade by intravenous propranolol and atropine. A selection criterion for the subjects of this part of the study was that the intrinsic sinus cycle length after autonomic blockade was as long or longer than the baseline spontaneous sinus length. This selection criterion allowed for atrial pacing after autonomic blockade at a cycle length equal to the baseline sinus cycle length. In this way, differences in the heart rate prior to the onset of ventricular pacing could be eliminated as a variable that might affect the measurement of ventricular refractory periods.

\section{Subjects of Study}

Forty-one subjects were recruited from among a pool of patients undergoing a clinicallyindicated electrophysiologic test. Selection criteria included a spontaneous sinus cycle length more than $600 \mathrm{~ms}$ and the lack of inducible ventricular tachycardia during programmed ventricular stimulation with one to three extrastimuli. Exclusion criteria consisted of: (1) atrial fibrillation; (2) current treatment with an antiarrhythmic drug or beta-adrenergic blocking agent; (3) New York Heart Association functional class 3 or 4 congestive heart failure; (4) angina pectoris or evidence of myocardial ischemia on a stress test; 
and (5) a fall in systolic arterial pressure to less than $85 \mathrm{mmHg}$ during continuous ventricular pacing at a cycle length of 600 or $400 \mathrm{~ms}$. An additional exclusion criterion was the inability to obtain two consecutive ventricular effective refractory period determinations within $5 \mathrm{~ms}$ of each other using a conventional extrastimulus technique (Method A); five patients were excluded because of this criterion.

The subjects consisted of 28 men and 13 women, and their mean age was $54 \pm 17$ years ( \pm one standard deviation). Eight patients had coronary artery disease, six patients had hypertension, three patients had a dilated cardiomyopathy, and 24 patients had no evidence of structural heart disease. The mean left ventricular ejection fraction as determined by contrast or radionuclide ventriculography was $0.52 \pm 0.08$. The clinical indication for the electrophysiologic test was evaluation of unexplained syncope in 24 patients, nonsustained ventricular tachycardia in ten patients, and paroxysmal supraventricular tachycardia in seven patients.

\section{Electrophysiologic Study Protocol}

Electrophysiologic studies were performed in the fasting, unsedated state after informed consent was obtained and at least 5 half-lives after discontinuation of therapy with antiarrhythmic and beta-adrenergic blocking agents. Depending on the clinical indication for the electrophysiologic study, two or three quadripolar electrode catheters were inserted into a femoral vein and positioned in the right atrium, across the tricuspid valve, or against the right ventricular apex. A short 5 French cannula inserted into a femoral artery was used to monitor the arterial pressure. Leads $V_{1}, I$, and III and the intracardiac electrograms were displayed on an osciloscope and recorded at a paper speed of $100 \mathrm{~mm} /$ second on a Siemens-Elema Mingograf-7 recorder (SiemensPacesetter, Inc., Sylmar, CA, USA). Programmed stimulation was performed with a programmable stimulator (Bloom Associates, Ltd.) using stimuli that had a duration of $2 \mathrm{~ms}$ and an intensity of twice diastolic threshold. The distal pair of electrodes of the quadripolar catheters was used for bipolar pacing and the proximal pair for recording the intracardiac electrograms.

\section{Measurement of Refractory Periods}

The study protocol was approved by the Human Research Committee at the University of Michigan Medical Center and was performed upon completion of the clinically-indicated portion of the electrophysiologic study. A quadripolar electrode catheter was positioned under fluoroscopic guidance at a stable pacing site in the right ventricular apex where the pacing threshold was $0.8 \mathrm{~mA}$ or less. In patients who had atrioventricular dissociation during ventricular pacing, a second electrode catheter positioned within the right atrium was used to pace the atrium simultaneously with the ventricle, to allow continuous ventricular pacing without interruption by sinus capture beats.

Method A for determination of the ventricular effective and functional refractory periods consisted of the use of 8 beat drive trains and a 4 second pause between drive trains (Fig. 1). Basic drive cycle lengths of 600 and $400 \mathrm{~ms}$ were used because they are representative of the basic drive cycle lengths commonly used in clinical electrophysiologic studies. ${ }^{13.15,19-24}$ The ventricular extrastimulus was initially positioned at a coupling interval of 200 to $220 \mathrm{~ms}$, which always was shorter than the ventricular effective refractory period. The extrastimulus coupling interval was increased in steps of $5 \mathrm{~ms}$ up to a coupling interval $10 \mathrm{~ms}$ longer than the extrastimulus coupling interval at which a ventricular response was first elicited. Each coupling interval was repeated to assure reproducible capture or noncapture. The effective refractory period was defined as the longest extrastimulus coupling interval that reproducibly failed to evoke a ventricular depolarization and the functional refractory period was defined as the shortest measured interval between the depolarizations resulting from the last basic drive stimulus and the extrastimulus. Method A was repeated in order to ascertain that the reproducibility of the effective refractory period was within $5 \mathrm{~ms}$.

Method B for determination of the ventricular effective and functional refractory periods consisted of 3 minutes of continuous pacing at the basic drive cycle length followed by the introduction of an extrastimulus after every eighth basic drive beat (Fig. 1). When there was ventricular capture by the extrastimulus, a pause equal to 


\section{METHOD A}

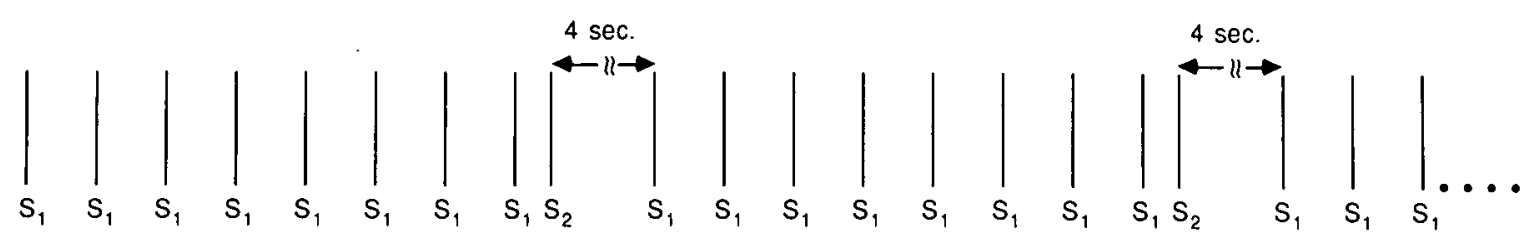

\section{METHOD B}

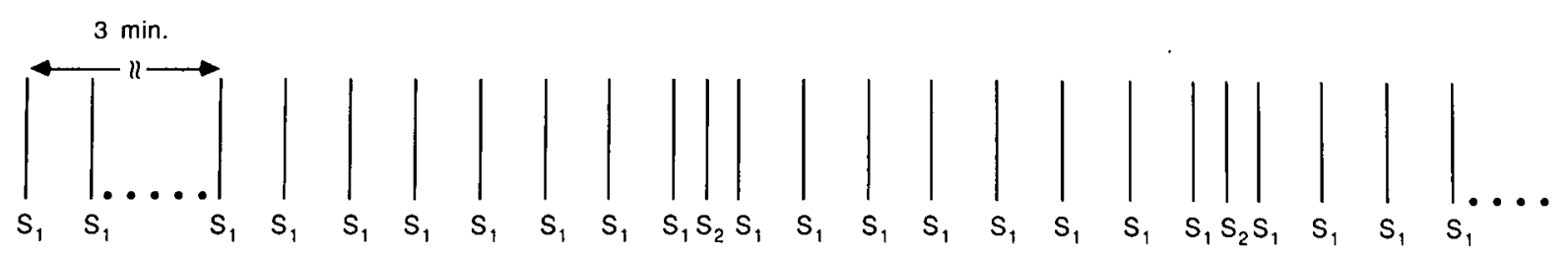

Figure 1. Methods $A$ and $B$ for determination of ventricular refractory periods. In Method $A$, the basic drive trains $\left(S_{1}\right)$ were 8 beats in duration and there was a 4 second intertrain pause. In Method $B$, continuous pacing was performed for 3 minutes before insertion of an extrastimulus $\left(S_{2}\right)$ after every eighth $S_{1}$ and initially there was no pause after the $S_{2}$ In both Methods $A$ and $B$, the initial $S_{1} S_{2}$ coupling interval was shorter than the ventricular effective refractory period and the $\mathrm{S}_{1} \mathrm{~S}_{2}$ interval was increased in steps of $5 \mathrm{~ms}$ until ventricular capture occurred. In Method $\mathrm{B}$, when $S_{2}$ resulted in ventricular capture, a pause equal to the basic drive cycle length was inserted after $S_{2}$.

the basic drive cycle length was inserted after the extrastimulus. The initial extrastimulus coupling interval was 170 to $200 \mathrm{~ms}$, which always was shorter than the ventricular effective refractory period. The extrastimulus coupling interval was then increased in steps of $5 \mathrm{~ms}$ and the refractory periods were determined as in Method A. The use of an initial extrastimulus coupling interval shorter than the effective refractory period avoided the recurrent, abrupt changes in rate that accompany the use of an extrastimulus started at a coupling interval longer than the effective refractory period.

In 12 subjects the ventricular effective refractory period was measured twice by Method $\mathrm{B}$ at a basic drive cycle length of $600 \mathrm{~ms}$ (six subjects) or $400 \mathrm{~ms}$ (six subjects), on one occasion with an initial pacing duration of 3 minutes, and on the next occasion with an initial pacing duration of 10 minutes. The two determinations in each subject were separated by at least a 2 minute rest period.

In 23 subjects, the ventricular refractory periods were measured by both Methods A and B.
The duration of pacing required to obtain the maximum shortening in ventricular refractory periods then was determined in the same 23 subjects. Continuous ventricular pacing at the basic drive cycle length was instituted and an extrastimulus was inserted every fourth beat. The extrastimulus coupling interval was fixed at an interval $5 \mathrm{~ms}$ longer than the effective refractory period as determined in that subject by Method $B$. Pacing was continued at the basic drive cycle length until the extrastimulus evoked a ventricular response, and the number of basic drive beats preceding ventricular capture by the extrastimulus was counted. To quantitate the variability in the number of basic drive beats required before ventricular capture by an extrastimulus set at a coupling interval $5 \mathrm{~ms}$ longer than the ventricular effective refractory period as determined by Method B, this step of the protocol was performed three times. Each of the three determinations was preceded by at least a 2 minute rest period. This procedure was performed first using a basic drive cycle length of $600 \mathrm{~ms}$, then $400 \mathrm{~ms}$. 


\section{Autonomic Blockade}

In six subjects, ventricular refractory periods were measured by Methods $A$ and $B$ before and after the intravenous administration of $0.2 \mathrm{mg} / \mathrm{kg}$ of propranolol followed immediately by 0.04 $\mathrm{mg} / \mathrm{kg}$ of atropine. These dosages of propranolol and atropine previously have been demonstrated to result in autonomic blockade in human. ${ }^{25}$ Both drugs were administered at a rate of $1 \mathrm{mg} / \mathrm{min}$ ute. The propranolol loading dose was followed by a continuous infusion of $0.2 \mathrm{mg} /$ minute in an attempt to maintain a steady plasma propranolol concentration during the testing interval. Testing was commenced 5 minutes after the administration of the propranolol loading dose. After autonomic blockade, the atria were continuously paced at a cycle length equal to the baseline spontaneous cycle length before autonomic blockade.

The plasma propranolol concentration was measured by high performance liquid chromatography with fluorescence detection. ${ }^{26}$ The plasma propranolol concentration was measured immediately before and after the testing interval in each of the six subjects, and the mean concentrations were $104 \pm 9 \mathrm{mcg} / \mathrm{L}$ and $93 \pm 5 \mathrm{mcg} / \mathrm{L}$, respectively. With the assay used, the range of plasma propranolol concentrations associated with beta adrenergic blockade is 50 to $100 \mathrm{mcg} / \mathrm{L}$.

\section{Analysis of Data}

A paired $t$-test was used to compare refractory periods determined after 3 versus 10 minutes of continuous pacing, refractory periods determined by Method A versus Method B, and the number of basic drive beats before ventricular capture at a basic drive cycle length of $600 \mathrm{~ms}$ versus $400 \mathrm{~ms}$

The three determinations of the number of basic drive beats before ventricular capture were compared using a repeated measures analysis of variance. Because the difference between the three determinations was not statistically significant, the three determinations were averaged for further analysis. The relationship between the spontaneous cycle length and the difference in refractory periods determined by Methods $A$ and $B$ was determined using Pearson's correlation coefficient, as was the relationship between change in cycle length and the number of basic drive beats before ventricular capture.

The effects of autonomic blockade on the refractory period determinations by Methods $A$ and $B$ were analyzed using a repeated measures analysis of variance. Multiple comparisons were performed using Fisher's least significant difference procedure. A p value less than 0.05 was considered significant.

\section{Results}

\section{Refractory Periods after 3 and 10 Minutes of Pacing}

The mean ventricular effective refractory period determined by Method $B$ at a basic drive cycle length of $600 \mathrm{~ms}$ in six subjects was $238 \pm 13$ ms when the duration of the basic drive train was 3 minutes and remained unchanged at $238 \pm 14$ $\mathrm{ms}$ when the duration of the basic drive train was increased to 10 minutes. The mean ventricular effective refractory period determined at a basic drive cycle length of $400 \mathrm{~ms}$ in six subjects also did not differ significantly when the basic drive train duration was 3 minutes as compared to 10 minutes $(198 \pm 17 \mathrm{~ms}$ and $199 \pm 15 \mathrm{~ms}$, respectively).

\section{Refractory Periods Measured by Methods $A$ and $B$}

The mean ventricular effective and functional refractory periods determined in 23 subjects by Method $B$ were significantly shorter than the corresponding determinations by Method A, both at a basic drive cycle length of 600 and 400 $\mathrm{ms}(\mathrm{p}<0.0001$, Table I). The mean difference between the effective refractory periods determined by the two methods at a basic drive cycle length of $400 \mathrm{~ms}, 33 \pm 9 \mathrm{~ms}$, was significantly larger than the mean difference between the two methods at a basic drive cycle length of $600 \mathrm{~ms}, 12 \pm 7 \mathrm{~ms}$ ( $p$ $<0.0001$ ).

There was a significant correlation between the spontaneous cycle length and the difference in effective refractory periods determined by Methods $A$ and $B(r=0.71 ; p=0.001$ at basic drive cycle length $600 \mathrm{~ms} ; \mathrm{r}=0.46, \mathrm{p}=0.05$ at basic drive cycle length $400 \mathrm{~ms}$; Fig. 2). 
Table I.

Mean Ventricular Refractory Periods Determined by Methods A \& B in 23 Subjects

\begin{tabular}{lllll}
\hline & Method A & Method B & A - B* & A vs. B (P Value) \\
\hline VERP (msec) & & & &. \\
BDCL 600 ms & $251 \pm 15^{* *}$ & $238 \pm 17$ & $12 \pm 7$ & $<0.0001$ \\
BDCL 400 ms & $231 \pm 14$ & $198 \pm 16$ & $33 \pm 9$ & $<0.0001$ \\
VFRP (msec) & & & & \\
BDCL 600 ms & $274 \pm 19$ & $258 \pm 17$ & $16 \pm 10$ & $<0.0001$ \\
BDCL 400 ms & $252 \pm 14$ & $218 \pm 12$ & $33 \pm 9$ & $<0.0001$ \\
\hline
\end{tabular}

- Difference between Methods A and B.

** Mean \pm standard deviation.

Abbreviations: $\mathrm{BDCL}$ = basic drive cycle length; VERP = ventricular effective refractory period; VFRP = ventricular functional refractory period.

\section{Effect of Automonic Blockade}

The mean spontaneous cycle length was 858 $\pm 168 \mathrm{~ms}$ in the baseline state and increased to $952 \pm 158 \mathrm{~ms}$ after autonomic blockade in the six patients who received propranolol and atropine $(p$ $<0.05$ ). The individual effective refractory period determinations are listed in Table 2. Autonomic blockade had no significant effect either on the mean effective refractory periods or on the magnitude of the difference between Methods A and $B$.

\section{Drive Train Duration Required for Maximum Shortening of Refractoriness}

The individual determinations of the number of basic drive beats required before capture by an extrastimulus set at a coupling interval $5 \mathrm{~ms}$ longer than the ventricular effective refractory period as determined by Method $B$ are presented in Table 3. The average spread between the highest and lowest values among the three determinations was 38 beats at a basic drive cycle length of $600 \mathrm{~ms}$ and 56 beats at a basic drive cycle length of $400 \mathrm{~ms}$. For each basic drive cycle length, the means of the three determinations did not differ from each other $(p=0.45$ and 0.36 at basic drive cycle lengths of $600 \mathrm{~ms}$ and $400 \mathrm{~ms}$, respectively).

The mean number of basic drive beats before ventricular capture at a basic drive cycle length of $400 \mathrm{~ms}, 233 \pm 85$ beats, was significantly greater than at a basic drive cycle length of $600 \mathrm{~ms}, 114$ \pm 84 beats, $(p<0.001)$.
BDCL 600 msec.

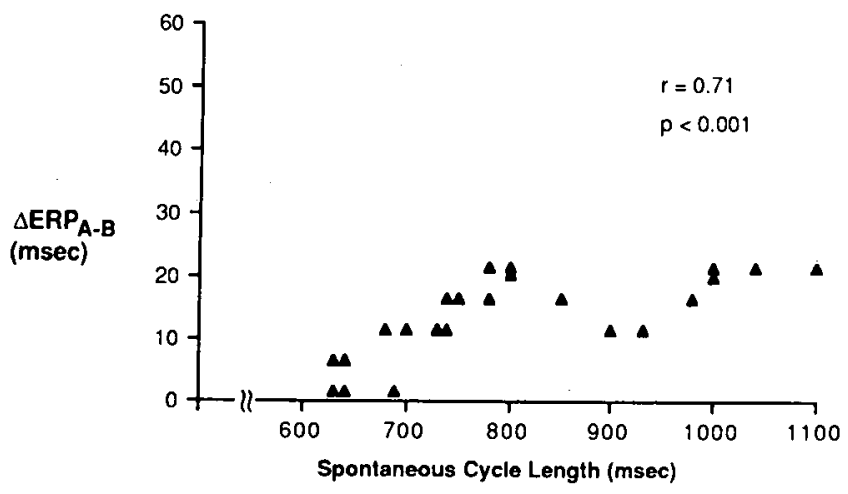

BDCL 400 msec.

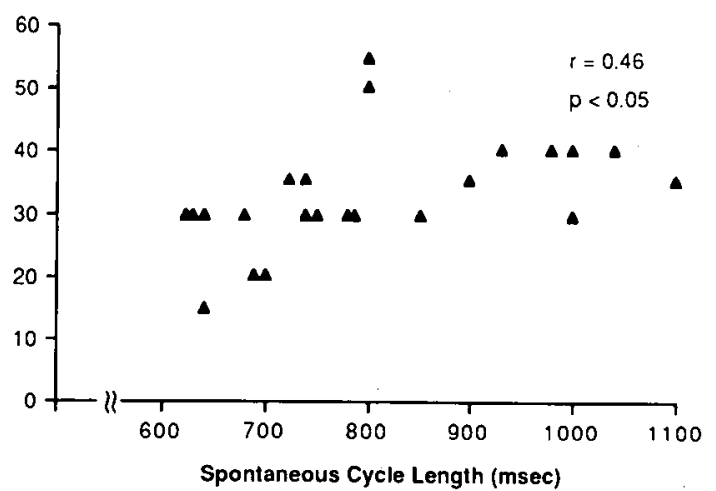

Figure 2. Correlation between the spontaneous cycle length and the amount of shortening in ventricular effective refractory period with Method $B$ as compared to Method $A\left(E R P_{A-B}\right)$ in 23 subjects. The correlation was significant at a basic drive cycle length (BDCL) of both 600 and $400 \mathrm{~ms}$. 
Table II. Ventricular Effective Refractory Periods Before and After Autonomic Blockade in Six Subjects

\begin{tabular}{|c|c|c|c|c|c|c|}
\hline \multirow[b]{2}{*}{ Subject } & \multicolumn{3}{|c|}{ Baseline VERP's (msec) } & \multicolumn{3}{|c|}{$\begin{array}{l}\text { VERP's After Autonomic Blockade } \\
\text { (msec) }\end{array}$} \\
\hline & $\begin{array}{l}\text { Method } \\
\text { A }\end{array}$ & $\begin{array}{l}\text { Method } \\
\text { B }\end{array}$ & $\mathbf{A}-\mathbf{B}^{*}$ & Method A & Method B & $\mathbf{A}-\mathbf{B}^{*}$ \\
\hline \multicolumn{7}{|c|}{ Basic Drive Cycle Length $600 \mathrm{msec}$} \\
\hline 36 & 230 & 215 & 15 & 235 & 215 & 20 \\
\hline 37 & 250 & 235 & 15 & 255 & 235 & 20 \\
\hline 38 & 275 & 250 & 25 & 285 & 265 & 20 \\
\hline 39 & 265 & 240 & 25 & 260 & 240 & 20 \\
\hline 40 & 255 & 240 & 15 & 260 & 245 & 15 \\
\hline 41 & 260 & 245 & 15 & 265 & 250 & 15 \\
\hline Mean $\pm S D$ & $256 \pm 15$ & $238 \pm 12$ & $18 \pm 15$ & $260 \pm 16^{* *}$ & $242 \pm 17^{\star \star}$ & $18 \pm 3^{\star \star}$ \\
\hline \multicolumn{7}{|c|}{ Basic Drive Cycle Length $400 \mathrm{msec}$} \\
\hline 36 & 210 & 195 & 15 & 215 & 190 & 25 \\
\hline 37 & 230 & 200 & 30 & 240 & 200 & 40 \\
\hline 38 & 255 & 210 & 45 & 265 & 255 & 40 \\
\hline 39 & 240 & 210 & 30 & 235 & 210 & 25 \\
\hline 40 & 230 & 195 & 35 & 240 & 200 & 40 \\
\hline 41 & 240 & 205 & 35 & 250 & 210 & 40 \\
\hline Mean $\pm S D$ & $234 \pm 15$ & $202 \pm 7$ & $32 \pm 10$ & $241 \pm 16^{\star *}$ & $206 \pm 12^{* *}$ & $35 \pm 8^{* *}$ \\
\hline
\end{tabular}

- Difference between Methods A and B.

** Not significantly different than corresponding baseline value.

Abbreviations as in Tables 1 and 2.

Combining the results obtained at the two basic drive cycle lengths, the difference between the spontaneous cycle length and the basic drive cycle length was related directly to the number of basic drive beats required for maximum shortening of the ventricular effective refractory period at the corresponding basic drive cycle length ( $\mathrm{r}$ $=0.62, \mathrm{p}<0.002$; Fig. 3).

\section{Discussion}

\section{Cumulative Effect of Basic Drive Train on Ventricular Refractoriness}

The results of this study demonstrate that ventricular muscle refractoriness in humans is highly dependent on the duration of the basic drive train. Depending on the baseline spontaneous cycle length and the basic drive cycle length, the ventricular effective refractory period measured after 3 minutes of continuous pacing may often be 20 to $40 \mathrm{~ms}$ shorter than when measured in a conventional manner with 8 beat basic drive trains and a 4 second intertrain pause. The cumulative effect of the basic drive train on ventricular refractoriness appears to be at its maximum by 3 minutes of pacing, with no further shortening of the refractory periods when the duration of the basic drive train is increased to 10 minutes.

\section{Mechanism of Cumulative Effect on Ventricular Refractoriness}

The cumulative effect of prolonged pacing on human ventricular refractory periods following an increase in rate may be explained by the experimental in vitro observation that the ventricular muscle action potential duration shortens progressively for 1 to 2 minutes before reaching a new steady state duration after an increase in the stimulation rate. ${ }^{1,6-10}$

An additional consideration in humans is the possible role of the autonomic nervous system as a factor influencing ventricular refractory pe- 
Table III.

Three Determinations of the Number of Basic Drive Beats Required for Maximum Shortening of Ventricular Refractoriness at Basic Drive Cycle Lengths of 600 and $400 \mathrm{msec}$

\begin{tabular}{|c|c|c|c|c|c|c|}
\hline \multirow[b]{2}{*}{ Subject } & \multicolumn{3}{|c|}{ No. of $\mathrm{S}_{1}$ 's at BDCL $600 \mathrm{msec}^{*}$} & \multicolumn{3}{|c|}{ No. of $\mathrm{S}_{1}$ 's at BDCL 400 msec* } \\
\hline & $\# 1$ & $\# 2$ & \#3 & $\# 1$ & \#2 & $\# 3$ \\
\hline 13 & 212 & 244 & 228 & 312 & 344 & 372 \\
\hline 14 & 152 & 72 & 96 & 272 & 320 & 280 \\
\hline 15 & 235 & 280 & 232 & 280 & 332 & 388 \\
\hline 16 & 88 & 124 & 84 & 232 & 240 & 208 \\
\hline 17 & 4 & 4 & 4 & 104 & 144 & 172 \\
\hline 18 & 100 & 120 & 128 & 160 & 192 & 124 \\
\hline 19 & 88 & 60 & 96 & 128 & 156 & 140 \\
\hline 20 & 4 & 8 & 4 & 76 & 76 & 100 \\
\hline 21 & 4 & 4 & 12 & 300 & 268 & 276 \\
\hline 22 & 80 & 140 & 128 & 128 & 72 & 64 \\
\hline 23 & 232 & 216 & 184 & 268 & 280 & 244 \\
\hline 24 & 132 & 212 & 120 & 280 & 336 & 252 \\
\hline 25 & 20 & 12 & 8 & 368 & 356 & 372 \\
\hline 26 & 16 & 16 & 16 & 112 & 188 & 200 \\
\hline 27 & 132 & 152 & 124 & 108 & 104 & 124 \\
\hline 28 & 176 & 276 & 248 & 276 & 180 & 288 \\
\hline 29 & 172 & 128 & 140 & 160 & 188 & 184 \\
\hline 30 & 108 & 68 & 80 & 224 & 248 & 208 \\
\hline 31 & 152 & 172 & 164 & 276 & 252 & 320 \\
\hline 32 & 4 & 4 & 4 & 212 & 180 & 240 \\
\hline 33 & 68 & 88 & 48 & 272 & 276 & 320 \\
\hline 34 & 256 & 260 & 300 & 364 & 268 & 320 \\
\hline 35 & 140 & 84 & 100 & 328 & 288 & 340 \\
\hline Mean $\pm S D$ & $112 \pm 80$ & $119 \pm 94$ & $111 \pm 86$ & $228 \pm 88$ & $230 \pm 85$ & $241 \pm 93$ \\
\hline
\end{tabular}

- For each basic drive cycle length, the means of the 3 determinations did not differ significantly $(p>0.05)$. Abbreviations: $S_{1}=$ basic drive beat; other abbreviations as in Table 2 .

riods. Continuous pacing at a cycle length of 600 or $400 \mathrm{~ms}$ often may be associated with a fall in blood pressure and therefore presumably sympathetic activation or vagal inhibition. Because sympathetic stimulation and vagal inhibition both result in a decrease in ventricular refractory periods, ${ }^{27-31}$ it could be argued that the shortening in ventricular refractoriness that occurs when the basic drive train is 3 minutes is a result of fluctuations in autonomic tone instead of being related to a prolonged duration of pacing required to obtain a steady-state action potential duration.

A comparison of ventricular refractory periods before and after autonomic blockade by propranolol and atropine demonstrated that the cumulative effect of 3 minutes of pacing on ventricular refractoriness was not attenuated by autonomic blockade. Therefore, it can be concluded that the decrease in ventricular refractory periods that occurs with prolonged pacing is not a result of sympathetic activation. However, an effect of vagal withdrawal cannot be ruled-out.

\section{Duration of Pacing Required to Obtain Maximum Shortening of Refractoriness}

This study has demonstrated that the duration of pacing required to obtain the maximum shortening of ventricular refractoriness is highly variable, ranging from 4 to 388 beats. The number 


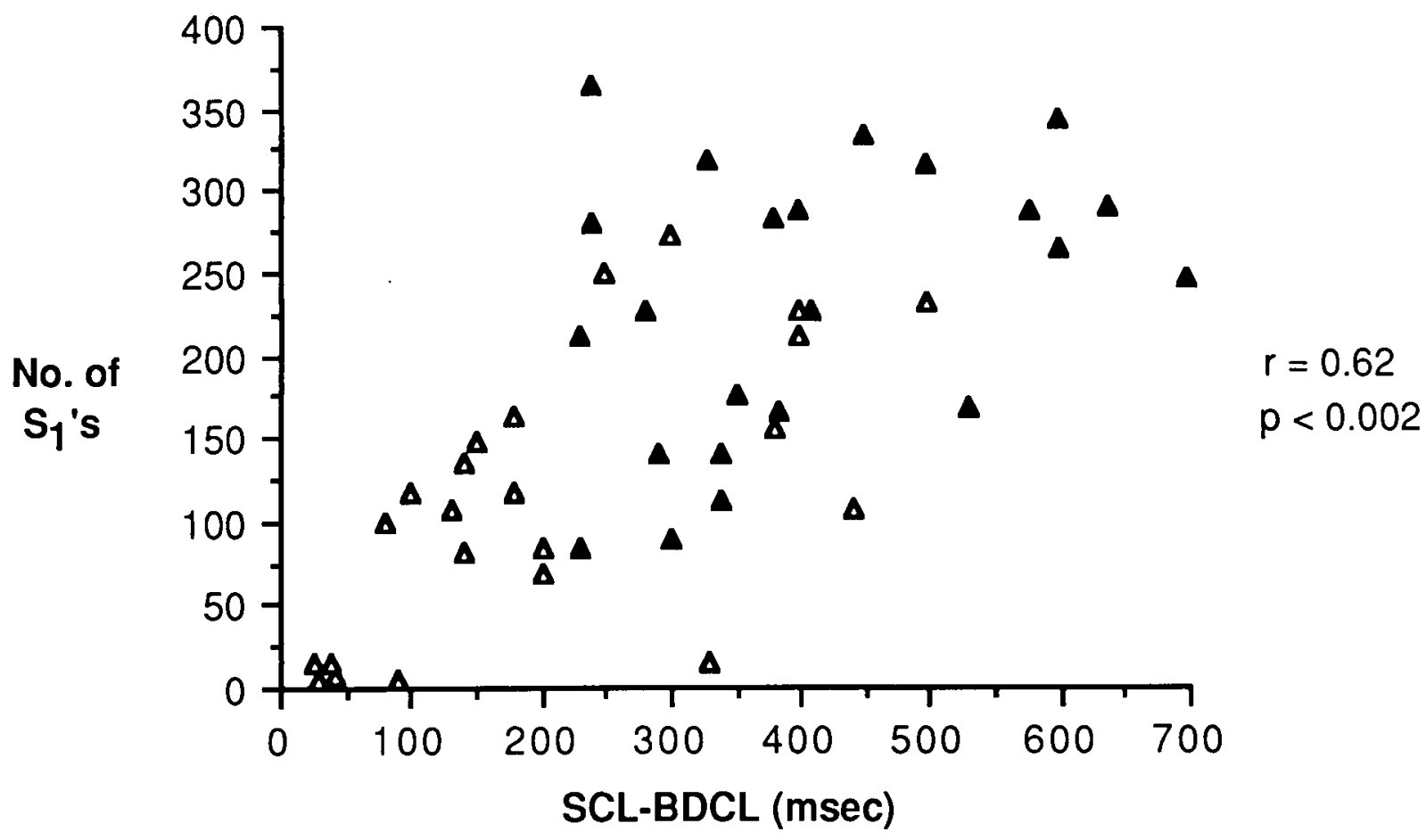

Figure 3. Correlation between change in rate (spontaneous cycle length [SCL] minus basic drive cycle length $[B D C L]$ ) and the number of basic drive beats $\left(S_{1}\right)$ required for maximum shortening of the ventricular effective refractory period in 23 subjects. The number of $S_{1} S^{\prime}$ 's required for maximum shortening of the ventricular effective refractory period was determined in triplicate, and each data point in this graph represents a mean of the three determinations. In each patient, measurements were made at a BDCL of both $600 \mathrm{~ms}$ (open triangles) and $400 \mathrm{~ms}$ (closed triangles).

of basic drive beats required to obtain the maximum effect of an increase in rate was dependent at least in part on the difference between the baseline spontaneous cycle length and the basic drive cycle length. This suggests that the number of basic drive beats required to reach a steadystate action potential duration and maximum shortening in ventricular refractoriness after an increase in rate may be directly related to the magnitude of the change in rate.

The large degree of inter-patient variability in the number of basic drive beats needed to achieve a maximum shortening of ventricular refractoriness is not entirely explained by differences in baseline spontaneous cycle length, as manifest by the relatively weak correlation between these two variables $(r=0.62)$. This observation indicates that there may be significant inter-patient variability in the time course of adaptation of ventricular refractoriness to an increase in rate. It should be noted that stimulation was performed only at a current strength of twice the late diastolic threshold and that the variability may have been less had higher current strengths been used.

There was also significant intra-patient variability in the number of basic drive beats needed to achieve the maximum shortening of refractoriness following an increase in rate. The reason for this variability is unclear. Of note is that the variability among the three determinations at each basic drive cycle length in each patient was random, with no significant difference between the means of the three determinations. This indicates that the variability was not caused by a systematic bias in the study design.

\section{Comparison with Prior Studies}

In an in situ canine preparation, Janse et al. studied the time course of shortening of ventricular refractoriness following an abrupt doubling in 
pacing rate. ${ }^{5}$ These investigators demonstrated that the maximal shortening in ventricular refractoriness develops gradually. When the basic drive cycle length was decreased from 600 to 300 $\mathrm{ms}, 400$ to 500 basic drive beats were required before a steady state refractory period was reached. When the final basic drive cycle length was 400,500 , or $600 \mathrm{~ms}, 160$ to 240 basic drive beats were required to reach a steady state refractory period. The results obtained at basic drive cycle lengths of 400 and $600 \mathrm{~ms}$ are comparable to the results obtained at the same basic drive cycle lengths in the present study.

Wiener et al. demonstrated that human ventricular refractoriness is dependent on the drive train duration, however drive trains only up to 8 beats in duration were evaluated. ${ }^{32}$ When there was a sudden decrease in cycle length, a single drive beat at the shorter interval produced 60 percent of the shortening of the refractory period produced by 8 drive beats at the shorter interval.

Only two preliminary reports have investigated the changes in ventricular refractoriness that occur with long basic drive trains. Prystowsky and Miles found that the mean ventricular effective refractory period decreased by $20 \mathrm{~ms}$ at a basic drive cycle length of $600 \mathrm{~ms}$ and by $28 \mathrm{~ms}$ at a basic drive cycle length of $400 \mathrm{~ms}$ as the drive train duration increased from 2 to 50 beats. ${ }^{11}$ The values obtained with a drive train duration of 8 beats were not reported. Brownstein et al. reported that the ventricular effective refractory period decreased by a mean of $11 \mathrm{~ms}$ as the drive train duration increased from 1 to 7 beats, and by an additional $18 \mathrm{~ms}$ as the drive train duration increased from 7 to 50 beats. ${ }^{12}$ The mean basic drive cycle length in the latter report was $327 \mathrm{~ms}$. Because of differences in study design, the results of these two studies cannot be compared in a quantitative fashion to the results of the present study.

\section{Limitations}

There are two limitations in the design of the study. Firstly, for reasons of patient safety, an alpha-adrenergic blocking agent was not administered to the subjects who received propranolol and atropine and, therefore, autonomic blockade in these subjects was not complete. Because un- opposed alpha-adrenergic stimulation has been demonstrated to result in mild prolongation of ventricular refractoriness in humans, ${ }^{33}$ sympathetic activation during 3 minutes of ventricular pacing in subjects who are beta-blocked possibly could result in an increase in ventriculàr refractory periods. Therefore, the maximum effect of an increase in rate on ventricular refractoriness may have been underestimated in this study.

A second limitation of the study design has to do with the technique used to determine the number of basic drive beats required to obtain the maximum shortening of ventricular refractoriness following an increase in rate. Because the end point of this technique was ventricular capture by an extrastimulus at a coupling interval that was $5 \mathrm{~ms}$ longer than the minimum effective refractory period, the technigue underestimated the actual number of basic drive beats required to reach the minimum effective refractory period. For example, if the extrastimulus had been set at a coupling interval only $1 \mathrm{~ms}$ longer than the minimum effective refractory period, the number of basic drive beats before ventricular capture by the extrastimulus most likely would have been greater. Ideally, the drive train duration required to obtain the maximum shortening of ventricular refractoriness would have been determined by repeatedly scanning with an extrastimulus to the point of refractoriness following progressively longer drive train durations. However, the length of time required for this process would be prohibitive for a clinical study.

Another limitation of this study is that only two basic drive cycle lengths were used and, therefore, the findings cannot be applied in a quantitative fashion to basic drive cycle lengths longer than $600 \mathrm{~ms}$ or shorter than $400 \mathrm{~ms}$. For example, it is possible that a drive train duration longer than three minutes may be required to achieve the maximum shortening in ventricular refractoriness at a basic drive cycle length shorter than $400 \mathrm{~ms}$.

\section{Conclusions}

Depending on the spontaneous cycle length and the basic drive cycle length, more than 200 basic drive beats often are required before the maximum effect of an increase in rate on ventric- 
ular refractoriness is achieved. Therefore, the conventional technique used to measure ventricular refractory periods with eight beat basic drive trains often may result in a substantial overestimate of the actual ventricular effective refractory period. This does not necessarily negate the value of conventional effective refractory period measurements as relative indicators of the effects of interventions such as antiarrhythmic drugs. However, it remains to be determined whether antiar-

\section{References}

1. Gibbs CL, Johnson EA. Effect of changes in frequency of stimulation upon rabbit ventricular action potential. Circ Res 1981; 9:165-170.

2. Mendez C, Gruhzit C, Moe G. Influence of cycle length upon refractory period of auricles, ventricles and A-V node in the dog. Am J Physiol 1956; 184:287-295.

3. Denes $\mathrm{P}, \mathrm{Wu} \mathrm{D}$, Dhingra $\mathrm{R}$, et al. The effects of cycle length on cardiac refractory periods in man. Circulation 1974; 49:32-41.

4. Guss S, Kastor J, Josephson M, et al. Human ventricular refractoriness. Circulation 1976; 53:450456.

5. Janse MJ, van der Steen ABM, van Dam RTh, et al. Refractory period of the dog's ventricular myocardium following sudden changes in frequency. Circ Res 1969; 24:251-262.

6. Vick RL. Action potential duration in canine Purkinje tissue: Effects of preceding excitation. J Electrocardiol 1971; 4:105-115.

7. Miller JP, Wallace AG, Feezor MD. A quantitative comparison of the relation between the shape of the action potential and the pattern of stimulation in canine ventricular muscle and Purkinje fibers. J Mol Cell Cardiol 1971; 2:3-19.

8. Boyett MR. An analysis of the effect of the rate of stimulation and adrenaline on the duration of the cardiac action potential. Pfluger Archiv 1978; 377:155-166.

9. Lab MJ, Yardley J. Electrophysiological differences between apex and base in intact pig ventricle. J Physiol 1979; 292:62P-63P.

10. Boyett MR, Jewell BR. Analysis of the effects of changes in rate and rhythm upon electrical activity in the heart. Prog Biophysiol Mol Biol 1980; 36:1-52.

11. Prystowsky EN, Miles WM. Human ventricular refractoriness: Interdependency of drive train duration, current strength and heart rate. (abstract) J Am Coll Cardiol 1984; 3:537.

12. Brownstein SL, Bauernfeind R, Welch $W$, et al. Cumulative effects of cycle length on ventricular refractoriness in man. (abstract) PACE 1987; $10: 432$.

13. Livelli FD, Jr., Bigger JT, Feiffel JA, et al. Response rhythmic drugs alter the time course of changes in ventricular refractoriness following an increase in rate. If so, this would imply that conventional effective refractory period determinations may be inaccurate even as relative indicators of drug effects on ventricular refractoriness.

Acknowledgments: The authors gratefully acknowledge the technical assistance of Beverly Burgie, Joan Bergeron, and Linda Abbott, and the secretarial assistance of Lisa Hackbarth.

to programmed ventricular stimulation: Sensitivity, specificity and relation to heart disease. Am J Cardiol 1982; 50:452-458.

14. Swiryn S, Bauernfeind RA, Strasberg B, et al. Prediction of response to class I antiarrhythmic drugs during electrophysiologic study of ventricular tachycardia. Am Heart J 1982; 104:43-50.

15. DiMarco JP, Garan H, Ruskin JN. Quinidine for ventricular arrhythmias: Value of electrophysiologic testing. Am J Cardiol 1983; 51:90-95.

16. Marchlinski FE, Buxton AE, Vassallo JA, et al. Comparative electrophysiologic effects of intravenous and oral procainamide in patients with sustained ventricular arrhythmias. J Am Coll Cardiol 1984; 4:1247-1254.

17. Estes NAM, Garan H, McGovern B, et al. Influence of drive cycle length during programmed stimulation on induction of ventricular arrhythmias: Analysis of 403 patients. Am J Cardiol 1986; 57:108-112.

18. Morady F, DiCarlo LA, Jr., de Buitlier M, et al. Effects of incremental doses of procainamide on ventricular refractoriness, intraventricular conduction and induction of ventricular tachycardia. Circulation 1986; 74:1355-1364.

19. Naccarelli GV, Prystowsky EN, Jackman WM, et al. Role of electrophysiologic testing in managing patients who have ventricular tachycardia unrelated to coronary artery disease. Am J Cardiol 1982; 50:165-171.

20. Denker S, Lehmann M, Mahnud R, et al. Facilitation of ventricular tachycardia induction with abrupt changes in ventricular cycle length. Am I Cardiol 1984; 53:508-515.

21. Buston $\mathrm{AE}$, Waxman HL, Marchlinski FE, et al. Role of triple extrastimuli during electrophysiologic study of patients with documented sustained ventricular tachyarrhythmias. Circulation 1984; 69:532-540.

22. Brugada P, Wellens HJJ. Comparison in the same patient of two programmed ventricular stimulation protocols to induce ventricular tachycardia. Am J Cardiol 1985; 55:380-383.

23. Herre JM, Mann DE, Luck JC, et al. Effect of increased current, multiple pacing sites and number 
of extrastimuli on induction of ventricular tachycardia. Am J Cardiol 1986; 56:102-107.

24. Morady F, DiCarlo LA, Jr., Baerman JM, et al. Comparison of coupling intervals that induce clinical and nonclinical forms of ventricular tachycardia during programmed stimulation. Am J Cardiol 1986; 57:1269-1273.

25. Jose AD, Taylor RR. Autonomic blockade by propranolol and atropine to study intrinsic myocardial function in man. J Clin Invest 1969; 48:20192031.

26. Schneck DW, Pritchard JF, Hayes AH. Measurement of propranolol, 4-hydroxypropranolol, and propranolol glycol in human plasma. Res Commun Chem Pathol Pharmacol 1979; 24:3-12.

27. Reddy CP, Gettes LS. Use of isoproterenol as an aid to electric induction of chronic recurrent ventricular tachycardia. Am J Cardiol 1979; 44:705713.

28. Freedman RA, Swerdlow CD, Echt DS, et al. Facilitation of ventricular tachyarrhythmia induction by isoproterenol. Am J Cardiol 1984; 54:765-770.
29. Olshansky B, Martins JB. Usefulness of isproterenol facilitation of ventricular tachycardia induction during estrastimulus testing in predicting effective chronic therapy with beta-adrenergic blockade. Am J Cardiol 1987; 59:573-577.

30. Prystowsky EN, Jackman WM, Rinkenberger RL, et al. Effect of autonomic blockade on ventricular refractoriness and atrioventricular nodal conduction in humans. Evidence supporting a direct cholinergic action on ventricular muscle refractoriness. Circ Res 1981; 49:511-518.

31. Morady F, Kou WH, Nelson SD, et al. Accentuated antagonism between beta-adrenergic and vagal effects on ventricular refractoriness in humans. Circulation 1988; 77:289-297.

32. Wiener I, Kunkes S, Rubin D, et al. Effects of sudden change in cycle length on human atrial, atrioventricular nodal and ventricular refractory periods. Circulation 1981; 64:245-248.

33. Morady F, Nelson SD, Kou WH, et al. The electrophysiologic effects of epinephrine in humans. J Am Coll Cardiol 1988; 11:1235-44. 
This document is a scanned copy of a printed document. No warranty is given about the accuracy of the copy. Users should refer to the original published version of the material. 